\title{
THREE STUDIES IN HIGHER CATEGORY THEORY: FIBRATIONS, SKEW-MONOIDAL STRUCTURES AND EXCISION OF EXTREMALS
}

\author{
MITCHELL BUCKLEY
}

(Received 4 November 2015; first published online 21 July 2016)

2010 Mathematics subject classification: primary 18D10; secondary 18D05, 18D30.

Keywords and phrases: fibration, bicategory, fibred category, fibred bicategory, Catalan simplicial set, monoidal category, skew-monoidal category, parity complex, excision of extremals.

Many fundamental constructions from ordinary category theory can be generalised to higher categories. Obvious examples include adjunctions, monads, algebras, limits and colimits. This thesis explores three cases where a construction from category theory is extended to higher categories.

We first consider the theory of (Grothendieck) fibrations and the Grothendieck construction. Our goal is to generalise this theory to the contexts of 2-categories and bicategories. Previous authors had made significant steps in this direction $[1,6]$ but attempts at a full description were incomplete or contained errors. The ordinary Grothendieck construction describes a correspondence between fibrations over a fixed category $B$ and contravariant pseudo-functors from $B$ into the 2-category of categories. By examining contravariant 2-functors from a 2-category $B$ into the 3-category of 2-categories, we are able to define a very strict kind of fibration for 2-categories. Following this, we examine contravariant trihomomorphisms from a bicategory $B$ into the tricategory of bicategories and obtain a very general notion of fibration for bicategories. Fibrations of bicategories exhibit many of the usual properties of ordinary fibrations. They are defined by asking that a given homomorphism have 'cartesian' lifts of certain 1-cells and 2-cells (where the usual notion of cartesian is suitably adjusted), they are closed under composition and a strict kind of bicategorical pullback, their cartesian 1-cells are defined by a bipullback and free fibrations can be formed by building a particular comma bicategory. The main result is the Grothendieck construction which presents a correspondence between fibrations of bicategories and contravariant trihomomorphisms into the tricategory of bicategories. This material has been published in [2].

Thesis submitted to Macquarie University in June 2015; degree approved on 5 August 2015; principal supervisor Dominic Verity, associate supervisor Richard Garner.

(c) 2016 Australian Mathematical Publishing Association Inc. 0004-9727/2016 \$16.00 
We next consider skew-monoidal categories. Our goal is to uncover a definition of skew-monoidal bicategory (a definition which is nontrivial due to the absence of a coherence theorem for skew-monoidal categories). We do this by introducing the Catalan simplicial set $\mathbb{C}$ and show that simplicial maps from $\mathbb{C}$ into an appropriate nerve of Cat are precisely skew-monoidal categories. This classifying property can be extended: simplicial maps from $\mathbb{C}$ into an appropriately chosen nerve can be monads, monoids, pseudo-monoids, skew-monoids, monoidal categories or monoidal bicategories. By examining simplicial maps from $\mathbb{C}$ to a suitable nerve of Bicat, we obtain a definition of skew-monoidal bicategory that is consistent with existing definitions of monoidal bicategory. The simplicial set $\mathbb{C}$ is itself uniquely isomorphic to the monoidal nerve of the poset $2=\perp \leqslant T$ (seen as a monoidal category under disjunction) and has a number of interesting combinatorial properties. This material has been published in [5] and [4].

Finally, we consider Ross Street's work on parity complexes [7]. Parity complexes are multi-dimensional graph-like objects that exhibit the minimal structure required to build free $n$-categories such as the orientals. Due to its detailed combinatorial nature, the material in this paper can be difficult to follow and quite hard to verify. Indeed, there are minor errors in the original text that were later corrected [8]. We present a formalisation, in Coq, of this theory up to the excision of extremals algorithm in Section 4. We have verified that Street's work is fundamentally sound and that there are no further errors. We summarise the main content of the theory and the basic intuition involved in its construction. We also discuss some technical aspects of the formalisation, and comment on which portions of the theory could benefit from some refinement. This material has been published in [3].

\section{References}

[1] I. Baković, 'Fibrations of bicategories', http://www.irb.hr/korisnici/ibakovic/groth2fib.pdf, 2012.

[2] M. Buckley, 'Fibred 2-categories and bicategories', J. Pure Appl. Algebra 218(6) (2014), 1034-1074.

[3] M. Buckley, 'A formal verification of the theory of parity complexes', J. Formaliz. Reason. 8(1) (2015), 1-24.

[4] M. Buckley, 'The Catalan simplicial set II', Appl. Categ. Structures 23 (2015), 33 pages.

[5] M. Buckley, R. Garner, S. Lack and R. Street, 'The Catalan simplicial set', Math. Proc. Cambridge Philos. Soc. 158(2) (2015), 211-222.

[6] C. Hermida, 'Some properties of Fib as a fibred 2-category', J. Pure Appl. Algebra 134(1) (1999), 83-109.

[7] R. Street, 'Parity complexes', Cah. Topol. Géom. Différ. Catég. 32(4) (1991), 315-343.

[8] R. Street, 'Parity complexes: corrigenda', Cah. Topol. Géom. Différ. Catég. 35(4) (1994), 359-361.

\section{MITCHELL BUCKLEY,}

Département de Mathématique,

Université Libre de Bruxelles,

Bureau 2.O.8.206, Campus de la Plaine,

Boulevard du Triomphe, B-1050 Bruxelles, Belgium

e-mail: mitchell.alan.buckley@gmail.com 